\title{
Objective Measures of Behavior Manifestations in Adult ADHD and Differentiation from Participants with Bipolar II Disorder, Borderline Personality Disorder, Participants with Disconfirmed ADHD as Well as Normative Participants
}

\author{
Hanna Edebol ${ }^{1, *}$, Lars Helldin $^{2}$ and Torsten Norlander ${ }^{3,4}$ \\ ${ }^{I}$ Department of Psychology, Karlstad University, Karlstad, Sweden \\ ${ }^{2}$ Department of Psychiatry, the NU-health Care, Trollhättan, Sweden \\ ${ }^{3}$ Evidens Research and Development Center, Göteborg, Sweden \\ ${ }^{4}$ Division of Psychology, Department of Clinical Neuroscience, Karolinska Institutet, Solna, Sweden
}

\begin{abstract}
Background: The present study evaluated two psychometric instruments derived from the objective measurement of adult ADHD using the Quantified Behavior Test Plus. The instruments were examined in ADHD versus a clinical group with overlapping symptoms including borderline personality disorder and bipolar II disorder, and another clinical group with participants assessed for but disconfirmed a diagnosis of ADHD as well as adult normative participants.

Methods: The Quantified Behavior Test Plus includes Continuous Performance Testing and a Motion Tracking System with parameters related to attention and activity operationalized as the cardinal symptoms of ADHD and then summarized into a Weighed Core Symptoms scale with ten cut-points ranging from 0 to 100. A categorical predictor variable called Prediction of ADHD was used to examine the levels of sensitivity and specificity for the Quantified Behavior Test Plus with regard to ADHD.

Results: The Weighed Core Symptoms scale separated ADHD and normative participants from each other as well as from the two clinical reference groups. The scale reported highest levels of core symptoms in the ADHD group and the lowest level of core symptoms in the normative group. Analyses with Prediction of ADHD yielded $85 \%$ specificity for the normative group, $87 \%$ sensitivity for the ADHD group, $36 \%$ sensitivity for the bipolar II and borderline group and $41 \%$ sensitivity for the group with a disconfirmed diagnosis of ADHD.

Conclusions: The Weighed Core Symptoms scale facilitated objective assessment of adult ADHD insofar that the ADHD group presented more core symptoms than the other two clinical groups and the normative group. Sensitivity for the Quantified Behavior Test Plus was lower in complex clinical groups with Bipolar II disorder, Borderline disorder and in patients with a disconfirmed diagnosis of ADHD. The psychometric instruments may be further evaluated with regard to well-documented and effective treatment programs for ADHD core symptoms.
\end{abstract}

Keywords: Adults, Attention Deficit Hyperactivity Disorder, Behavior, Hyperactivity, Objective measures, Psychometrics, Quantified Behavior Test Plus.

\section{BACKGROUND}

A prevalence of about $4.4 \%$ in the general population makes Attention Deficit Hyperactivity Disorder (ADHD) one of the most commonly occurring adult psychiatric disorders [1]. Further findings [2] suggest that the occurrence of ADHD is highly comorbid with other psychiatric disorders, associated with life-long impairment, dysfunction in major areas of life and under-treatment. ADHD covers cardinal symptoms of hyperactivity, impulsivity and inattention that may be found alone or combined as described in the DSMIV [3]. Core behaviors of ADHD are also common characteristics of other psychiatric disorders [4] and examples of common disorders with symptom-overlap are mood and

*Address correspondence to this author at the Department of Psychology, Karlstad University, Karlstad, Sweden; Tel: (0046) 54700-1145;

Fax: (0046) 5483 9165; E-mail: hanna.edebol@kau.se anxiety, other neuropsychiatric and personality disorders of borderline and antisocial character [5, 6]. Bipolar disorders for example often include the attention symptom domain such as low attention span, forgetfulness and distractibility as well as the activity symptom domain with increased psychomotor activity, impulsive and intrusive behaviors as well as affective symptoms of irritability, emotional lability and tantrum outbursts which may be found in ADHD as well [7, 8]. The clinical significance of these behaviors is subjectively evaluated with regard to differential diagnoses and comorbid disorders as well as the level of manifestation and malfunctioning in daily life using rating scales, interviews and sometimes neuropsychological tests [4]. However, it is widely recognized that behaviors of ADHD are demanding to discriminate from over-lapping and co-existing psychiatric conditions when the assessments are based on qualitative and unstandardized tools alone $[4,9,10]$. 
Cognitive symptoms in particular are not unique for ADHD as inattention and impulsivity are universal symptoms of adult behavior disorders that offer few diagnostic directions [11-13]. Research findings suggest that a more objective symptom of ADHD is hyperactivity which is especially significant for ADHD in the combined hyperactive/impulsive and inattentive form [14]. Even though hyperactivity is not present in all subjects with ADHD, peripheral motor activity such as fidgeting are commonly self-reported and confirmed by independent observers of adults with the disorder $[15,16]$. Studies $[17,18]$ using objective measures of ADHD core symptoms report that adults with the disorder are substantially more active than healthy controls, and that objective measures of hyperactivity such as motion tracking systems and actigraphs are more effective in discriminating between ADHD and community controls than performance testing of attention and impulsivity. Similar results of marked hyperactivity in ADHD are found in both male and female subjects but findings suggest that females diagnosed with ADHD present more psychomotor activity than males with ADHD. The same is reversed for the community controls so that males in general are more active during attention-tasks than females [18]. Thus, previous research findings with objective measurement techniques suggest that hyperactivity persists into adulthood and may be a more distinct behavior of the disorder than symptoms of inattention and impulsivity. However, these studies $[17,18]$ also suggest that objective measures of the actual activity level may differ from that of subjective evaluation and clinical impression since hyperactivity is often overlooked and mistaken for other behavior disorders.

A well-used objective measurement technique for ADHD is the continuous performance test (CPT). The overarching aim of these computerized tests is to quantify the amount of impulsive and inattentive behaviors by means of appropriate and reliable measures such as commission and omission errors. Behaviors of impulsivity and inattention make up two thirds of the ADHD-diagnostic criteria and have been wellinvestigated with the CPT in psychiatric research [19]. Studies throughout the years generally support the utility of CPTs in adult ADHD [10, 20,21] and meta-analyses suggest that especially measures of inattention have been fruitful among adults $[22,23]$. Improvements in attention and activity have been well-documented with the CPT and motion tracking systems when comparing stimulant pretreatment and onmedication conditions [24-28] as well as with alternative treatment approaches such as neurofeedback, EEG [29, 30]. Interestingly, a meta-analysis of EEG [31] using various outcome measures including the CPT and self-reports found large effects sizes for inattention and impulsivity as well as medium effect sizes for hyperactivity. Neurofeedback has also been found to be a helpful diagnostic tool for childhood ADHD and specificity in clinical comparison groups typically range from $60-90 \%$ [30]. Even though no comparable EEG studies have been collected for adults with ADHD at this time, the approach presents comparable results as the computerized tools with promising clinical utility in childhood ADHD and deserves future studying. The CPT paradigm have been frequently applied in the assessment and treatment of ADHD but three general problems have been discussed, 1) the CPT paradigm has been critized to lack sensitivity for adult ADHD and especially in those cases with less severe symptomatology [32, 33], 2) it has been increasingly recognized that traditional CPTs do not separate ADHD from other clinical groups [34-36] and 3), the CPT lack psychometric measures of hyperactivity which is needed to evaluate ADHD and therapeutic interventions. Previous studies $[37,38]$ investigating CPT measures combined with a motion tracking system reported two new psychometric instruments for adult ADHD called the Weighed Core Symptoms (WCS) scale and Prediction of ADHD (PADHD), both derived from the Quantified Behavior Test Plus, QbTest-Plus [39, 40]. The instruments presented initial satisfactory psychometric properties but the results of WCS and PADHD have yet not been examined with regard to clinical groups with symptom overlap.

The present study aims to investigate the validity of WCS and PADHD in adults with ADHD as well as in clinical groups with overlapping symptoms, e. i., borderline and bipolar II disorders, participants assessed for but disconfirmed a diagnosis of ADHD and adult normative participants. In line with previous findings, it is predicted that participants with ADHD will show more symptoms than participants with bipolar II disorder, borderline disorder and those with a disconfirmed ADHD-diagnosis, and even more symptoms than normative participants.

\section{Hypothesis of the present study:}

Participants with ADHD have more ADHD-core symptoms than participants with bipolar II disorder, borderline disorder and participants with a disconfirmed diagnosis of ADHD, and even more core symptoms than normative participants without ADHD when assessed with PADHD and WCS.

\section{METHODS}

\section{Participants}

This study consisted of 306 participants belonging to four groups of participants: (a) having been diagnosed with Attention Deficit Hyperactivity Disorder (ADHD, $N=53$ ), (b) having either Bipolar II Disorder or Borderline Personality Disorder (Bipolar/Borderline, $N=45$ ), (c) having been assessed for but disconfirmed a diagnosis of ADHD (Disconfirmed, $N=29$ ), or (d) belonging to the adult normative group (Normative, $N=179$ ).

The ADHD Group. The mean age for participants in the ADHD group, including 24 men and 29 women, was 35.89 years $(S D=12.25$, range $=18$ to 64$)$. Participants $(n=50)$ were assessed at the clinic in neuropsychiatry and CBT, Cereb, or at the neuropsychiatric clinic in the NU-health care $(n=3)$, Sweden, at the mean age of 35.89 years $(S D=12.6$ range $=18$ to 64$)$ and diagnosed with ADHD according to the Diagnostic and Statistical Manual of Mental Disorders [3]. Most of the participants had the combined hyperactive/impulsive and inattentive subtype of ADHD (314.01; $n=47)$ while others had the predominantly inattentive subtype $(314.00 ; n=2)$ or ADHD-not otherwise specified $(314.9, n=4)$ because of not obtaining information from significant others regarding early childhood. The assessments included clinical interviews, self-rating scales, information from relatives and psychological testing including the QbTest-Plus [39, 40]. Except for ADHD, nine participants 
Table 1. Demographic Data for Clinical Groups Sample Size (n) and Percentage (\%) of the Clinical Groups in the Present Study that fell into Each Categorical Measure of Demographic Characteristics

\begin{tabular}{|c|c|c|c|c|c|c|}
\hline & \multicolumn{2}{|c|}{ Disconfirmed } & \multicolumn{2}{|c|}{ BipolarII/Borderline } & \multicolumn{2}{|c|}{ ADHD } \\
\hline & $n$ & $\%$ & $n$ & $\%$ & $n$ & $\%$ \\
\hline $\begin{array}{c}\text { Marital status } \\
\text { Single (not married) } \\
\text { Married/spouse now } \\
\text { Divorced/separated }\end{array}$ & $\begin{array}{c}18 \\
10 \\
1\end{array}$ & $\begin{array}{c}62 \\
34 \\
3\end{array}$ & $\begin{array}{c}17 \\
23 \\
4\end{array}$ & $\begin{array}{c}38 \\
51 \\
9\end{array}$ & $\begin{array}{c}27 \\
20 \\
5\end{array}$ & $\begin{array}{c}51 \\
38 \\
9\end{array}$ \\
\hline $\begin{array}{l}\text { Living arrangements } \\
\text { Live alone } \\
\text { Live with spouse } \\
\text { Group home }\end{array}$ & $\begin{array}{c}16 \\
13 \\
0\end{array}$ & $\begin{array}{c}55 \\
45 \\
0\end{array}$ & $\begin{array}{c}15 \\
27 \\
2\end{array}$ & $\begin{array}{c}33 \\
60 \\
4\end{array}$ & $\begin{array}{c}32 \\
20 \\
0\end{array}$ & $\begin{array}{c}60 \\
38 \\
0\end{array}$ \\
\hline Employment & 11 & 38 & 12 & 27 & 31 & 58 \\
\hline $\begin{array}{l}\text { Highest education } \\
\text { High school } \\
\text { Senior high school } \\
\text { Graduate school }\end{array}$ & $\begin{array}{c}6 \\
20 \\
3\end{array}$ & $\begin{array}{l}21 \\
69 \\
10\end{array}$ & $\begin{array}{c}12 \\
28 \\
4\end{array}$ & $\begin{array}{c}27 \\
62 \\
9\end{array}$ & $\begin{array}{l}12 \\
30 \\
10\end{array}$ & $\begin{array}{l}23 \\
57 \\
19\end{array}$ \\
\hline
\end{tabular}

had one $(n=7)$ or two $(n=2)$ additional psychiatric disorders including dyslexia $(n=3)$, social phobia $(n=3)$, generalized anxiety disorder $(n=1)$, depression $(n=2)$, stress reaction $(n=1)$ and emotionally instable personality disorder $(n=1)$. At the day of the test, 16 participants used one or more medications including antidepressants $(n=12)$, anxiolytics $(n=9)$, neuroleptics $(n=4)$, and/or antiepileptics $(n$ $=2$ ). The approximate mean time from taking medication at the test was 319.72 minutes, i.e., 5.39 hours $(S D=101.08$ minutes). For demographic information see Table 1.

The Bipolar/Borderline Group. The mean age for participants in this group, including 13 men and 32 women, was 42.33 years $(S D=11.63$, range $=22$ to 60$)$. The group had Bipolar II disorder $(n=27)$ or Borderline Personality Disorder $(n=18)$ and received their diagnosis at the mean age of 40.60 years $(S D=12.05$, range $=21$ to 69$)$. The mean total Global Assessment of Functioning [41] closest in time to the study was $55.67(n=42, S D=9.82$, range $=40$ to 95$)$. A total of 13 participants had one or several additional diagnoses, including; psychological and behavioral disturbances because of substance use $(n=4)$, generalized anxiety disorder $(n=3)$, social phobia $(n=2)$, panic disorders $(n=1)$, mixed states of anxiety- and depression $(n=2)$, adaption disorder $(n=1)$, relapsing depression $(n=1)$, and two persons with borderline personality disorder also had bipolar disorders (e.i., 296.89 and 296.80). At the day of the test, 42 participants used antidepressants $(n=33)$, anti-psychotics $(n$ $=12$ ), antiepileptics $(n=16)$, neuroleptics $(n=12)$, anxiolytics $(n=16)$, barbitones $(n=15)$, antabuse $(n=1)$ and/or medication for Parkinson $(n=2)$.

The Disconfirmed group. The mean age for participants in this group, including13 men and 16 women, was 35.21 years $(S D=10.31$, range $=20$ to 54$)$. Their mean age when disconfirming ADHD was 33.45 years $(S D=10.33$, range $=$ 19 to 52$)$ and most of the clinical assessments $(n=22)$ concerned ADHD specifically while some also included a broader field of neuropsychiatric disorders $(n=7)$. The out- come included none $(n=8)$, one $(n=9)$ or two $(n=12)$ psychiatric diagnoses, including Aspergers syndrome $(n=6)$, dyslexia $(n=4)$, personality disorders $(n=4)$, borderline personality disorder $(n=1)$, bipolar disorder unspecified $(n=2)$, obsessive compulsive disorder $(n=1)$, posttraumatic stress disorder $(n=1)$, memory disorder unspecified ( $n=1)$, as well as secondary diagnoses of depression ( $n$ $=3)$, dyscalculia $(N=2)$, attention disorders unspecified $(n=$ $2)$, developmental coordination disorder $(n=1)$, tics $(n=1)$, social phobia $(n=1)$, dysmorphobia $(n=1)$, and mixed substance use disorder $(n=1)$. Of the participants, 19 did not use psychotropic medication at the time of the test while 10 used one $(n=6)$, two $(n=2)$, three $(n=1)$, or four $(n=1)$ medications, including antidepressants $(n=8)$, neuroleptics $(n=2)$, antiepileptics $(n=5)$, anxiolytics $(n=1)$, and/or medication for opium dependency $(n=1)$. The approximate mean time from using medication at the first minute of the test was 168 minutes $(S D=569.56, n=25)$.

The Normative Group. This group $(N=179)$ included 99 men and 80 women from the adult normal population whose mean age was 31.45 years $(S D=10.33$, range $=18$ to 53). Inclusion criterions were ages between 18 to 65 years of age, willingness to sign the informed consent and complete the study-specific procedures and the exclusion criteria was any known psychiatric diagnosis. ADHD-symptoms were measured with the adult ADHD self-report scale [42], for means and standard deviation see Table 2 . A majority of the participants in the normative group $(n=113)$ were students at Karlstad University or personnel from various companies in Karlstad, Sweden, and the remaining part $(n=66)$ were recruited from music organizations in Giessen, Germany.

\section{Design}

The independent variables of the present study were Group (norm, disconfirmed, bipolar II/borderline, ADHD), Gender (men, women) and Prediction of ADHD (no, yes). There were 179 participants in the normative group, 53 participants in the ADHD-group, 45 participants in the bipolar 
Table 2. Descriptive data for ASRS

Means (M) and Standard Deviations (SD) for the Adult ADHD Self-Report Scale (ASRS) Total Score, ASRS Items of Hyperactivity and Impulsivity, ASRS Items of Inattention, and the Six Most Predictive Items of ASRS Reported for the Groups of the Present Study

\begin{tabular}{|c|c|c|c|c|c|c|c|c|}
\hline & \multicolumn{2}{|c|}{ Normative } & \multicolumn{2}{c|}{ Disconfirmed } & \multicolumn{2}{c|}{ BipolarII/Borderline } & \multicolumn{2}{c|}{ ADHD } \\
\hline & M & SD & M & SD & M & SD & M & SD \\
\hline \hline ASRS-Total & 22.70 & 6.88 & 36.86 & 11.74 & 36.38 & 11.75 & 45.42 & 11.33 \\
\hline Hyperactive/Impulsive & 10.53 & 3.88 & 16.64 & 6.17 & 17.05 & 6.44 & 21.06 & 5.67 \\
\hline Inattention & 12.17 & 4.02 & 21.08 & 6.98 & 19.79 & 6.22 & 24.13 & 7.12 \\
\hline ASRS-Predictive & 8.06 & 2.72 & 13.59 & 4.33 & 12.60 & 4.38 & 15.90 & 4.78 \\
\hline
\end{tabular}

II/borderline group and 29 participants in the disconfirmed group. The total number of men was 149 and the total number of women was 157. Dependent variables were ADHD core symptoms of hyperactivity, inattention, and impulsivity as well as the Weighed Core Symptoms (WCS) scale.

\section{Instruments}

QbTest-Plus. This instrument [39, 40] combines an XXtype Continuous Performance Test (CPT) installed as a software program on a PC and an activity test during 20 minutes. While performing the CPT on the computer, movements of the participants are recorded using an infrared Motion Tracking System (MTS) following a reflective marker attached to a head-band. The CPT involves rapid presentations of stimuli involving color (blue, red) and shape (circle, square) on the screen and participants are instructed to press a hand-held button when stimuli subsequently repeats itself (a target) and not to press the button when stimuli varies relative to the previous one (a non-target). The stimuli are presented at a pace of one per two seconds, each one visible for 200 milliseconds, and the total number of stimuli is 600 , presented with a $25 \%$ target probability. The purpose of QbTest-Plus is to provide objective information regarding cardinal symptoms of ADHD; hyperactivity on basis of motor-activity measured with the camera, and inattention and impulsivity on basis of the CPT-test [40].

Operationalization of variables was done according to a previous study [38]. Hyperactivity was operationalized with the parameter called "distance", i.e., the length (meter) of the path describing the movement of the headband reflector during the test. Inattention was operationalized as omission errors, that is when no response is registered and the stimulus was a target and finally, impulsivity was operationalized as commission errors and that is when a response is registered and the stimulus was a non-target. Former studies of QbTestPlus [17, 37, 38] demonstrate a relative significance of core symptoms with measures of hyperactivity being the most and inattention the second most efficient measure of ADHD whereas the level of impulsivity was the least efficient measure of ADHD as compared to healthy normative controls.

Weighed Core Symptoms Scale (WCS). This scale summarizes the total level of ADHD core symptoms in adulthood on a scale with ten cut-points ranging from 0 to 100 [38] where 0 indicate maximal amount of ADHD symptoms and 100 indicate complete absence of ADHD symptoms. The WCS scale is based upon raw scores from the summed and operationalized measures from the QbTest-Plus in which the results of hyperactivity has been multiplied with three, inattention with two and impulsivity with one. The ten cut-points of WCS have been developed through a procedure described by Edebol et al. [38]. In the present study, WCS correlated (Pearsons' $r$ ) with hyperactivity $(r=0.82, p<$ $0.001)$, inattention $(r=0.73, p<0.001)$, and impulsivity $(r=$ $0.50, p<0.001)$ and those results were about the same also when correlations were computed for the ADHD group or the normative group only.

Prediction of ADHD (PADHD). This categorical predictor variable [38] regarding ADHD (no, yes) is based upon raw scores from the operationalized measures of the QbTestPlus (Q-scores), e. i., hyperactivity measured in distance, inattention measured with omission errors and impulsivity measured with commission errors. PADHD was developed independently from WCS using qualitative analyses and assessment trials in which the level of sensitivity and specificity was evaluated. PADHD has previously generated $86 \%$ sensitivity and $83 \%$ specificity in predicting ADHD and healthy normative participants.

Global Assessment of Functioning Scale (GAF). The Global Assessment of Functioning scale [41] estimates psychological, social and occupational functioning on a numeric continuum (0-100) of mental health and illness for adult persons. The GAF-scores presented in the current study are the scores reported in the psychiatric records closest in time to QbTest-Plus and they have been carried out by clinicians.

The Adult Self Report Scale for Adult ADHD v1.1 (ASRS). This self-report screening instrument [42, 43] is derived from the criterions of ADHD in DSM-IV. Part A include the 6 most predictive items while part B holds an additional 12 items, all rated on a five-point scale $(0=$ never, 1 $=$ rarely, $2=$ sometimes, $3=$ often; and $4=$ very often). Each item has a cut-off point of either 2 (sometimes) or 3 (often), and four or more items listed above cut-off in part A is used as a cut-off for ADHD [40]. The internal consistency (Cronbachs alpha, $\alpha$ ) for the patient-administered version is 0.88 [43].

\section{Procedure}

Karlstad University is participating in a research program regarding objective markers of ADHD. The study procedures was examined and approved by the Regional Ethical Review Board of Uppsala, Sweden in February 2008 (2008/110/2). 
Table 3. Effects of Prediction of ADHD

Effects of Prediction of ADHD (No, Yes) for the Normative, Disconfirmed, Bipolar II/Borderline, and ADHD Groups

\begin{tabular}{|c|c|c|c|c|}
\hline \multicolumn{5}{|c|}{ Prediction of ADHD } \\
\hline & No & & Yes & \\
\hline Group & Frequency & Percent & Frequency & Percent \\
\hline Normative & 152 & 85 & 27 & 15 \\
\hline Disconfirmed & 12 & 41 & 17 & 59 \\
\hline Bipolar II/Borderline & 16 & 36 & 29 & 64 \\
\hline ADHD & 7 & 13 & 46 & 87 \\
\hline
\end{tabular}

The Normative Group. In Karlstad, recruiting was performed using major companies in the fields of education, transportation and industry as well as among students at the University. In Giessen, recruiting was performed via the Cognitive Neuroscience Centre for Psychiatry using music organizations. For further details on experimental testing with QbTest-Plus in the normative group please see the procedures described for the ADHD group below since procedures for the two groups coincide.

The ADHD Group. At the neuropsychiatric clinic Cereb, nurses screened psychiatric records from 2008, i.e., this was the time when Cereb started using computerized records, and randomly selected 150 of approximately 400 patients who had done the QbTest-Plus during their clinical assessment, oversampling those with ADHD in combined form and without severe psychiatric comorbidity. Participants were contacted via mail three times from May to June in 2011. After resending the mail, participants received compensation to a value of $100 \mathrm{SEK}$ for participating in the study. At the division of psychiatry in the county council of Värmland, clinicians screened psychiatric records and recruited participants on mail and telephone. The patients diagnose and status was unknown to the researcher during the whole process of experimental testing and assessment. When doing the QbTest-Plus, instructions was given verbally and then by the means of a standardized video [39] presenting procedures of the test. A room with minimal visual and auditory stimuli was used for the test.

The Bipolar/Borderline Group. Diagnostic assessments had been carried out within two separate psychiatric clinics, the NU-Health Care in Västra Götaland, Sweden $(n=29)$, and the psychiatric division of the County Council of Värmland, Sweden $(n=16)$. Clinicians screened 586 psychiatric records and recruited participants on mail and telephone. The study-specific procedures were explained and information on withholding ones diagnostic and medical status from the researcher was pointed out in particular.

The Disconfirmed Group. Diagnostic assessments had been carried out within three separate psychiatric clinics, the NU-Health Care $(n=6)$, the psychiatric division of the County Council of Värmland $(n=6)$ and Cereb $(n=17)$. Clinicians screened psychiatric records and recruited participants who had been assessed for ADHD/ADD and/or neuropsychiatric disorders including ADHD/ADD but not received such diagnoses. Study-specific procedures were explained and information on withholding ones diagnostic and medical status from the researcher was pointed out in particular. At Cereb, 50 participants who had done QbTest-Plus during clinical assessment were randomly selected and contacted via regular mail three times from May to June in 2010.

\section{RESULTS}

\section{Prediction of ADHD}

Pearson Chi-Square with Group (normative, disconfirmed, bipolar II/borderline, ADHD) and Prediction of ADHD (No, Yes) showed a strong significant connection ( $p$ $<0.001$ ) between variables indicating a vast majority of No in the normative group and a vast majority of Yes in the clinical groups. Effects regarding sensitivity and specificity for the predictive variable of the present study are shown in Table 3.

\section{Prediction of ADHD and WCS}

A one-way ANOVA with Prediction of ADHD (no, yes) as independent variable and WCS as dependent variable yielded a significant effect for Prediction $[F(1,304)=$ 411.06, $p<0.001]$. Descriptive statistics showed that the Yes-group scored lower $(M=20.59, S D=19.71)$ than the No-group $(M=71.02, S D=22.11)$ with regard to WCS.

\section{Group and Gender Differences with Regard to Depend- ent Variables}

A Pillai's MANOVA (4 x 2 factorial design) was conducted using Group (normative, disconfirmed, bipolar II/borderline, ADHD) and Gender (men, women) as independent variables and Hyperactivity, Inattention, and Impulsivity as dependent variables. The analysis yielded significant effects for Group $\left(p<0.001, E_{t a}{ }^{2}=0.21\right.$, power $\left.>0.99\right)$ and for the interaction Group $\mathrm{x}$ Gender $\left(p=0.040, E t a^{2}=\right.$ 0.02 , power $=0.85)$ but not for Gender $(p>0.05)$. Results from the univariate F-tests regarding Group and the interaction Group x Gender are given below.

Core Symptoms. Univariate F-tests yielded significant effects with regard to Group for Hyperactivity $[F(3,298)=$ $95.10, p<0.001]$, Inattention $[F(3,298)=38.60, p<0.001]$, and for Impulsivity $[F(3,298)=9.61, p<0.001]$ were the ADHD group consistently scored lower compared to clinical and normative controls. Post-hoc testing (Tukey HSD, $5 \%$ level) indicated that the ADHD group scored lower on Hyperactivity $(M=26.95, S D=23.91)$ compared to the other groups and also lower with regard to Inattention $(M=36.52$, $S D=14.84$ ) than the Normative and the Disconfirmed group 
Table 4. Descriptive Data for Dependent Variables

Means (M) and Standard Deviations (SD) for Hyperactivity, Inattention, Impulsivity and the Weighed Core Symptoms (WCS) Scale with Regard to Group and Gender

\begin{tabular}{|c|c|c|c|c|c|c|c|c|}
\hline & \multicolumn{4}{|c|}{ Normative } & \multicolumn{4}{|c|}{ Disconfirmed } \\
\hline & $\mathbf{M}$ & SD & $\mathbf{M}$ & SD & $\mathbf{M}$ & SD & $\mathbf{M}$ & SD \\
\hline Hyperactivity & 74.20 & 13.12 & 72.67 & 13.01 & 63.61 & 22.14 & 57.74 & 26.82 \\
\hline Inattention & 61.08 & 15.30 & 55.61 & 13.29 & 48.17 & 9.14 & 47.62 & 14.16 \\
\hline \multirow[t]{4}{*}{ WCS } & 70.81 & 23.20 & 64.13 & 24.84 & 40.00 & 30.00 & 43.13 & 29.83 \\
\hline & \multicolumn{4}{|c|}{ Bipolar II/Borderline } & \multicolumn{4}{|c|}{ ADHD } \\
\hline & \multicolumn{2}{|c|}{ Men } & \multicolumn{2}{|c|}{ Women } & \multicolumn{2}{|c|}{ Men } & \multicolumn{2}{|c|}{ Women } \\
\hline & M & SD & M & SD & M & SD & M & $\mathrm{SD}$ \\
\hline WCS & 46.15 & 28.73 & 36.56 & 29.80 & 17.92 & 20.85 & 6.21 & 8.62 \\
\hline
\end{tabular}

Note: Higher values on dependent variables indicate lower levels of ADHD symptoms.

but not significantly lower than the Bipolar II/Borderline group $(M=38.62, S D=19.92)$. Furthermore, with regard to Impulsivity, the post-hoc test showed that the ADHD group scored lower $(M=57.74, S D=29.50)$ than the Normative group $(M=74.33, S D=14.92)$ but not significantly lower than the Disconfirmed group $(M=64.53, S D=25.10)$ or the Bipolar II/Borderline group $(M=62.28, S D=30.10)$. Moreover, the Normative group scored higher on Hyperactivity $(M=73.51, S D=13.10)$ and Inattention $(M=58.63$, $S D=14.66)$ as compared to all other groups and they scored higher than the ADHD and the Bipolar II/Borderline groups with regard to Impulsivity $(M=74.33, S D=14.92)$ but they were not more impulsive than the Disconfirmed group $(M=$ $64.53, S D=25.10)$. The Bipolar II/Borderline group and the Disconfirmed group did not differ with regard to Hyperactivity or Impulsivity but only with regard to Inattention ( $p=$ $0.05)$, were the Bipolar II/Borderline group scored lower $(M$ $=38.62, S D=19.92)$ than the Disconfirmed group $(M=$ 47.87, $S D=11.97)$.

Analysis for the interaction of Group and Gender yielded significant results for Impulsivity $[F(3,298)=3.55, p=$ $0.015]$ but not for Hyperactivity or Inattention ( $p s>0.05)$. Independent samples t-tests $(5 \%$ level) showed that there were no significant differences between the sexes with regard to Impulsivity in the Normative, Bipolar II/Borderline or the ADHD groups but men in the Disconfirmed group were more impulsive $(M=53.32, S D=27.64)$ than women in that same group $(M=73.63, S D=19.14 ; t(27)=-2.33, \mathrm{p}$ $<0.05)$. Means and standard deviations are reported in Table 4.

Weighed Core Symptoms. Since the WCS scale is a composite measure it had to be analyzed separately with a two-way ANOVA. Analyses yielded a significant effect for Group $[F(3,298)=75.68, p<0.001]$ but not for Gender or the interaction Group x Gender ( $p s>0.05)$. A post-hoc test
(Tukey-HSD, $5 \%$ level) showed that the ADHD group scored significantly lower than all other groups on WCS $(M=11.51$, $S D=16.34)$ and the Normative group scored significantly higher than all other groups on WCS $(M=67.82, S D=24.11)$. There was no significant difference between the Disconfirmed group $(M=41.73, S D=29.41)$, and the Bipolar II/Borderline group $(M=39.33, S D=29.50)$ with regard to WCS. Means and standard deviations are presented in Table 4.

\section{DISCUSSION}

The present study examined two objective measures of adult ADHD, e. i., WCS and PADHD, on basis of the hypothesis stating that participants with ADHD have more ADHD-core symptoms than participants with bipolar II disorder, borderline disorder and participants with a disconfirmed diagnosis of ADHD, and even more core symptoms than normative participants without ADHD when assessed with PADHD and WCS.

In line with a previous study [38], most of the ADHD and normative participants in the present study were correctly classified with PADHD as reflected in the $87 \%$ sensitivity and $85 \%$ specificity. Clinical specificity was $36 \%$ for the Bipolar II/Borderline group and $41 \%$ for the disconfirmed ADHD group which suggest that there are greater similarities between these groups than between the normative and the ADHD group with regard to core symptoms of ADHD. This lower clinical specificity makes sense from clinical perspectives since bipolar II/borderline and disconfirmed participants often presents symptoms of ADHD and especially cognitive symptoms, and the disconfirmed group has even been assessed for ADHD. However, a major difference between the bipolar II/borderline and the disconfirmed participants on the one hand and the ADHD participants on the other hand is the total level of behavior manifestations 
during the test and PADHD suggest that the total level of core symptoms are greater in ADHD versus both other clinical groups. Nevertheless, the meaningfulness of PADHD as a clinical instrument is related to both sensitivity and specificity since $100 \%$ sensitivity and $0 \%$ specificity would not really involve any meaningful information about ADHD. PADHD seems rather sensitive to ADHD and specific in discerning healthy normative participants but less effective in differentiating other clinical groups with core symptoms of ADHD. One weakness of PADHD is the inability to represent ADHD behaviors in continuous terms although higher levels of core symptoms in the ADHD group were represented as higher general level of sensitivity for this group. To separate ADHD from other clinical groups it seems like a good idea to look into the quantitative measurement of behavior.

The Weighed Core Symptoms scale generated consistent differences between the ADHD and all other groups as well as between the normative and all other groups. The scale suggest that participants with ADHD are more hyperactive, impulsive and inattentive than bipolar II, borderline and disconfirmed participants and even more so than the normative participants. Despite the overlapping core symptoms between ADHD and the other clinical cases of this study, WCS generated consistent differences between the groups. A continuous and composite instrument like the WCS seems to be fruitful when examining differences between ADHD and clinical groups with shared symptoms since the total level of symptoms constitute a major difference between these groups.

Similar to several other studies $[17,18,38]$ of objective measurement techniques for ADHD, the most characteristic feature of the disorder in the present study was hyperactivity which was possible to measure with a motion tracking system and a reflective headband during experimental conditions. Raw scores and psychometric instruments of the present study confirm that the level of subtle motor activity was highly marked in subjects with ADHD as compared to normative participants. Results also demonstrate that hyperactivity was the most objective measure of ADHD versus other clinical groups. The second most objective measure of ADHD was the level of inattention that separated ADHD from both normative and disconfirmed participants, but not from the bipolar II and borderline group as they were equally inattentive as the ADHD group. Finally, the third most characteristic feature of ADHD was impulsivity which separated normative participants from ADHD but the clinical groups were equally impulsive as the ADHD group. Core symptom measures alone did not identify a significant number of subjects with ADHD but the composite measure generated significant differences because, unlike the core symptom measures, WCS is calibrated with the entire clinical ADHD-phenomenon and the relative impact of behavior manifestations.

WCS did not generate significant differences between the bipolar II/borderline and the disconfirmed groups as they had equal levels of hyperactivity and impulsivity. Instead, the major difference between the clinical groups was that the bipolar II/borderline group was more inattentive than the rejected group during the test. The results suggest that inattention is a common feature of adult psychiatric disorders rather than a unique aspect of ADHD and inattention needs to be complemented with measures of hyperactivity to better distinguish ADHD among clinical groups. Although developed independently from PADHD, WCS was used to investigate the predictive ability of PADHD and a majority of negative predictions fell into the higher range of WCS while a majority of the positive predictions fell into the lower range of WCS. The results suggest that PADHD and WCS correspond well together and with ADHD and may be combined for optimal prediction since PADHD is not highly predictive of ADHD amongst other clinical cases with shared symptoms.

No gender differences were found in the present study. However, analyses yielded a group and gender interaction in the disconfirmed group since men was more impulsive than women in this group. Knowledge about objective measures of ADHD manifestations in adulthood and especially with regard to gender is not well explored. Subjective measures of ADHD made with rating scales and observational reports sometimes emphasize gender differences [44] but the general understanding is that gender is not a significant contribution to symptom display or severity of ADHD symptoms per see but that prevalence constitutes a major gender difference and that subjective methods may blur identification of ADHD as compared to more objective techniques [45, 46].

Barkley and colleagues [47] investigated the DSM-IV symptom list among ADHD, community and clinical control groups to compare the levels of sensitivity and specificity from interviews. As in our study, they found that males and females within the ADHD group did not differ in severity of symptom display, and they also found that the symptom list correctly classified a majority of ADHD and community participants. However, nearly half of the clinical group reported levels of ADHD symptoms that would exceed the threshold which illustrate the complexity of separating ADHD from other clinical cases. The most frequently endorsed ADHD symptoms by the clinical controls in their study was related to inattention and found in $82-87 \%$ of the clinical cases and in $97 \%$ of ADHD cases. They concluded that ADHD symptoms in general, and symptoms of inattention in particular, are rather common in clinical samples, whether representing ADHD or not. But as symptoms were not too common in the general community (about $10 \%$ ), ADHD symptoms above threshold is a strong marker of a mental disorder. Moreover, in the large UMASS study, Barkley and his colleagues [47] analyzed a broader pool of items that was related to ADHD core symptoms as well as other executive dysfunctions that is thought to underlie core symptoms of ADHD and found the similar patterns of more impairment in the ADHD than in the community group. The best results relative to clinical cases in the UMASS study were obtained when reducing the symptom list to identification of $65 \%$ of the ADHD cases and at the same time significantly $\left(X^{2}, 5 \%\right.$ alpha level) more likely to be presented in those cases than in the clinical control cases. The best classification accuracy however was reported for six items that were referred to as executive dysfunctions and resulted in $65 \%$ accuracy in the clinical control group and $85 \%$ in the ADHD group. But this means that, at best $65 \%$ of the clinical cases were identified as having ADHD which is 
cal cases were identified as having ADHD which is comparable to the results of PADHD and reflects the very complexity and commonness of ADHD-symptoms among clinical cases. Barkley [48] defines executive functions as three interrelated processes of behavioral inhibition, including inhibiting prepotent responses, stopping ongoing responses to create a period of delay and, protecting that period of delay from disruption of competing responses. One may think of the executive functions as more or less active during attention-tasks and reflected in behaviors of inattention, impulsivity and increased psychomotor activity, as presented by both the clinical and ADHD cases in the present study. Perhaps the executive functioning paradigm illustrates the general and inclusive mechanisms that underlie behavioral mechanisms of ADHD as well as other behavior disorders. The present study and studies with similar objective measurement techniques, such as the ADHD Quotient System [17, 18], suggest that hyperactivity is a highly distinctive marker for the objective measurement of adult ADHD. The present study also proposes that hyperactivity is the most objective marker relative to clinical cases. However, most of the ADHD cases in the present study did not have severe psychiatric comorbidity and the implication of the results of the present study therefore may not be representative for adults with severe comorbidity.

The effect of comorbid psychiatric disorders upon combined CPT performance and motor activity tracking systems was assessed by Glickman [49] in clinical samples of children with ADHD alone or ADHD plus major depressive disorder. The groups was possible to separate on basis of five out of six motion and attention measures respectively and the combined measurement correctly classified $84 \%$ of ADHD cases but sensitivity for detection of ADHD in comorbid cases was significantly reduced when adding tests of executive functioning (card-sorting task), which may suggest that executive functioning tasks are less distinctive than objective measures of core symptoms in ADHD with other psychiatric disorders. Studies $[50,51]$ using actometry in adults with ADHD and antisocial personality disorder reported higher frequencies of movements as compared to healthy controls, and akathisia patients was found [51] to generate similar movement patterns as patients with ADHD which was theorized to stem from their hypodopaminergic etiologies. Nevertheless, it seems like ADHD plus psychiatric comorbidity may impose additional challenges onto the objective measurement of adult ADHD and distinc-tive measures like hyperactivity are therefore especially encouraging.

The present study had several limitations. It was based upon the same normative sample as the previous study of WCS and PADHD [38], which may inflict validity constrains upon evaluation of the measures. Further, most participants had ADHD in combined form which may cause limitations upon the generalization of data. The group with ADHD was tested with the QbTest-Plus by their clinical contact as a part of the thorough neuropsychological assessment which may create sample-biases or restrain the generalization of the present study. However, WCS and PADHD presented marked improvements for the validity of the test as compared to the unstandardized test report used in clinical practice [39].

Moreover, the group with a disconfirmed diagnosis of ADHD is not homogenous by their clinical character but rather by the fact that they all had been suspected to have ADHD, systematically assessed for it but considered not to have it. Therefore, this group may be considered to belong to the general "grey-zone" in which many clinical characteristics that is likely to resemble ADHD may be found and the group is thoroughly described in the methods section. The bipolar II/borderline group may be considered a mixed group with overlapping symptoms of ADHD as well as other clinical characteristics, and no additional information was gained by separating the two subgroups. The reason why we separated the bipolar II/borderline group from the disconfirmed group is because of their different clinical characteristics, and the disconfirmed group is less homogenous and not constituted by a specific clinical condition, which is also reflected in objective measures of impulsivity. Interestingly, results indicate that the bipolar II/borderline group had equal levels of inattention and impulsivity as the ADHD group and the disconfirmed group had equal levels of inattention as the ADHD group. The fact that the ADHD group is outstanding from clinical cases only with regard to hyperactivity may however be due to their diagnostic subtype. Nevertheless, the separation of ADHD in combined form from bipolar II, borderline and the disconfirmed group is encouraging. One may add that the previous study in which WCS and PADHD were standardized (see the methods section) included more representative proportions of the ADHD- diagnostic subtypes but yielded equal results as the present study. Future studies may however be more attentive towards differences regarding clinical definitions on the one hand and operationalized and functional measures of behavior manifestations on the other hand. Finally, larger samples would be needed in order to refine the standardization of these measures.

Keeping these limitations in mind, the results did however confirm the hypothesis of the present study stating that participants with ADHD have more ADHD-core symptoms than other clinical cases, and even more core symptoms than normative cases without ADHD. Moreover, adults with ADHD were especially marked by their higher levels of hyperactivity as compared to the other groups. The psychometric instruments investigated in the present study, WCS and PADHD, may be further evaluated with regard to well-documented and effective treatment programs aimed for symptom alleviation and achievement of remission in adults with ADHD. Future studies will hopefully shed further light on the validity of PADHD and WCS and investigate their relation to other measurement techniques as to learn more about behavior manifestations and effective treatment of adult ADHD.

\section{CONFLICT OF INTEREST}

The authors declare that they have no conflict of interest.

\section{ACKNOWLEDGEMENTS}

This study was supported by grants from the County Council of Värmland (LiV), Sweden, and by unrestricted grants from Janssen-Cilag AB, Sollentuna, Sweden. 


\section{REFERENCES}

[1] Kessler RC, Adler L, Gruber MJ, Sarawate CA, Spencer T, van Brunt DL. Validity of the World Health organization adult ADHD self-report scale (ASRS) screener in a representative sample of health plan members. Int J Methods Psychiatr Res 2007; 16: 52-65.

[2] Kessler RC, Adler L, Barkley R, et al. The prevalence and correlates of adult ADHD in the United Sates: results from the national comorbidity survey replication. Am J Psychiatry 2006; 163: 71623.

[3] American Psychiatric Association. Diagnostic and Statistical Manual of Mental Disorders (DSM-IV). $4^{\text {th }}$ ed. Washington DC: American Psychiatric Pub. 2000.

[4] Murphy KR, Adler LA. Assessing attention-deficit/hyperactivity disorder in adults: Focus on rating scales. J Clin Psychiatry 2004; 65: 8-11.

[5] Biederman J, Faraone SV, Spencer T, et al. Patterns of psychiatric comorbidity, cognition, and psychosocial functioning in adults with attention deficit hyperactivity disorder. Am J Psychiatry 1993; 150: $1792-8$.

[6] Wilens TE, Biederman J, Faraone SV, Marleton MK, Westerberg D, Spencer TJ. Presenting ADHD symptoms, and comorbid disorders in clinically referred adults with ADHD. J Clin Psychiatry 2009; 40: 1557-62.

[7] Giedd JN. Bipolar disorder and attention-deficit/hyperactivity disorder in children and adolescents. J Clin Psychiatry 2000; 61: 31-4.

[8] Kent L, Craddock N. Is there a relationship between attention deficit hyperactivity disorder and bipolar disorder? J Affect Disord 2003; 73: 211-21.

[9] Weiss MD, Weiss JR. A guide to the treatment of adults with ADHD. J Clin Psychiatry 2004; 65: 27-37.

[10] Woods SP, Lovejoy DW, Ball JD. Neuropsychological characteristics of adults with ADHD: a comprehensive review of initial studies. Clin Neuropsychol 2002; 16: 12-34.

[11] Adler LA. Clinical presentations of adult patients with ADHD. J Clin Psychiatry 2004; 65: 8-11.

[12] Jackson B, Farrugia D. Diagnosis and treatment of adults with attention deficit hyperactivity disorder. J Couns Dev 1997; 75: 3129.

[13] Spencer TJ. ADHD treatment across the life cycle. J Clin Psychiatry 2004; 65: 22-6.

[14] Murphy K, Barkley RA. Attention deficit hyperactivity disorder in adults: comorbidities and adaptive impairments. Compr Psychiatry 1996; 37: 393-401.

[15] Downey K, Stelson F, Pomerleau O, Giordiani B. Adult attention deficit hyperactivity disorder: psychological test profiles in a clinical population. J Nerv Ment Dis 1997; 185: 32-8.

[16] Marshall P, Schroeder R, O'Brien J, et al. Effectiveness of symptom validity measures in identifying cognitive and behavioral symptom exaggeration in adult attention deficit hyperactivity disorder. Clin Neuropsychol 2010; 24: 1204-37.

[17] Lis S, Baer N, Stein-en-Nosse C, Gallhofer B, Sammer G, Kirsch P. Objective measurement of motor activity during cognitive performance in adults with attention-deficit/hyperactivity disorder. Acta Psychiatr Scand 2010; 122: 285-94.

[18] Polcari A, Fourligas N, Navalta C, Teicher MH. Hyperactivity persists in male and female adults with ADHD and remains a highly discriminative feature of the disorder. American Academy of Child and Adolescent Psychiatry 2010 Oct 26-31: Proceedings of the $57^{\text {th }}$ annual meeting; New York, USA. 2010.

[19] Epstein JN, Erkanli A, Conners CK, Klaric J, Costello JE, Angold A. Relations between continuous performance measures and ADHD behaviors. J Abnorm Child Psychol 2003; 31: 543-54.

[20] Hervey AS, Epstein JN, Curry JF. Neuropsychology of adults with attention deficit/hyperactivity disorder: a meta-analytic review. Neuropsychology 2004; 18: 485-503.

[21] Willcutt EG, Doyle AE, Nigg JT, Faraone SV, Pennington BF. Validity of the executive function theory of attentiondeficit/hyperactivity disorder: a meta-analytic review. Biol Psychiatry $2005 ; 57: 1336-46$

[22] Boonstra AM, Oosterlaan J, Sergeant JA, Buitelaar JK. Executive functioning in adult ADHD: a meta-analytic review. Psychol Med 2005; 35: 1097-108.
[23] Frazier TW, Demaree HA, Youngstorm EA. Meta-analysis of intellectual and neuro-psychological test performance in attentiondeficit/hyperactivity disorder. Neuropsychology 2004; 18: 543-55.

[24] Losier BJ, McGrath PJ, Klein RM. Error patterns on the continuous performance test in non-medicated and medicated samples of children with and without ADHD: A meta-analysis review. J Child Psychol Psychiatry 1996; 37: 971-87.

[25] Heiser P, Frey J, Smidt C, et al. Objective meaurement of hyperactivity, impulsivity, and inattention in children with hyperkinetic disorders before and after treatment with methylphenidate. Eur Child Adolesc Psychiatry 2004; 13: 100.

[26] Tabori-Kraft J, Sorensen M, Kerguard M, Dalsgaard S, Thomsen PH. Is MMAT (Quotient) useful for monitoring the effect of stimulants on hyperactivity and inattention? A brief report. Eur Child Adolesc Psychiatry 2007; 16: 347-351.

[27] Teicher M, Polcari A, McGreenery C. Utility of objective measures of activity and attention in the assessment of therapeutic response to stimulants in children with ADHD. J Child Adolesc Psychopharmacol 2008; 18: 265-270

[28] Teicher M, Lowen S, Polcari A, Foley M, McGreenery C. Novel strategy for the analysis of CPT data provides new insight into the effects of methylphenidate on attentional states in children with ADHD. J Child Adolesc Psychopharmacol 2004; 14: 2.

[29] Fuchs T, Birbaumer N, Luttzenberger W, Gruzelier JH, Kaiser J. Neurofeedback treatment for attention-deficit/hyperactivity disorder in children: a comparison with methylphenidate. Appl Psychophysiol Biofeedback 2003; 28: 1-12.

[30] Loo SK, Barkley RA. Clinical utility of EEG in Attention Deficit Hyperactivity Disorder. Appl Neuropsychol 2005; 12: 64-76.

[31] Arns M, Ridder S, Srehl U, Breteler M, Coenen A. Efficiacy of neurofeedback treatment in ADHD: the effects on inattention, impulsivity and hyperactivity: a meta-analysis. Clin EEG Neurosci 2009; 40: 180-9.

[32] Corbett B, Stanzcak DE. Neuropsychological performance of adults evidencing attention deficit hyperactivity disorder. Arch Clin Neuropsychol 1999; 14: 373-87.

[33] Seidman LJ, Biederman J, Faraone SV, Weber W, Ouellette C. Toward defining a neuropsychology of attention deficithyperactivity disorder: Performance of children and adolescents from a large clinically referred sample. J Consult Clin Psychol 1997; 65:150-60.

[34] Epstein JN, Johnson DE, Varia IM, Conners CK. Neuropsychological assessment of response inhibition in adults with ADHD. J Clin Exp Neuropsychol 2001; 23: 362-71.

[35] Katz LJ, Wood DS, Goldstein G, Auchenbach RC, Geckle M. The utility of neuropsychological tests in evaluation of AttentionDeficit/Hyperactivity Disorder (ADHD) in adults. Assessment 1998; 5: 45-51.

[36] Walker AJ, Shores EA, Trollor JN, Lee T, Sachdev PS. Neuropsychological functioning of adults with attention deficit hyperactivity disorder. J Clin Exp Neuropsychol 2000; 22: 115-24.

[37] Edebol H, Helldin L, Holmberg E, Gustafsson S, Norlander T. In search for an objective measure of hyperactivity, impulsivity and inattention in adult attention deficit hyperactivity disorder using the quantified behavior test plus. Eur J Psychol 2011; 7: 443-57.

[38] Edebol H, Helldin L, Norlander, T. Measuring adult attention deficit hyperactivity disorder using the quantified behavior test plus. Psychol J 2011 [In review].

[39] QbTech AB. QbTest Plus Clinical Manual. Gothenburg 2010a

[40] QbTech AB. QbTest Plus Technical Manual. Gothenburg 2010b.

[41] Luborsky L. Clinicians' judgments of mental health. Arch Gen Psychiatry 1962; 7: 407-17.

[42] Kessler RC, Adler L, Ames M, et al. The world health organization adult ADHD self-report scale (ASRS): a short screening scale for use in the general population. Psychol Med 2005; 35: 245-56.

[43] Kessler RC, Ustün TB. The world mental health (WMH) survey initiative version of the world health organization (WHO) composite international diagnostic interview (CIDI). Int J Methods Psychiatr Res 2004; 13: 93-121.

[44] Shaffer D. Attention deficit hyperactivity disorder in adults. Am J Psychiatry 1994; 151: 633-38.

[45] Arcia E, Conners KC. Gender differences in ADHD? J Dev Behav Pediatr 1998; 19: 77-83.

[46] Biederman J, Faraone SV, Monuteaux MC, Bober M, Cadogen E. Gender effects on attention-deficit/hyperactivity disorder in adults, revisited. Biol Psychiatry 2004; 55: 692-700. 
[47] Barkley RA, Murphy KR, Fischer M. ADHD in adults- what the science says. New York: The Guilford Press 2008.

[48] Barkley RA. Inhibition, sustained attention, and executive functions: constructing a unifying theory of ADHD. Psychol Bull 1997; 121: 65-94.

[49] Glickman M. Objective measures of motion, attention and executive function in the identification of attention deficit hyperactivity disorder among children with major depression [dissertation]. Dallas (TX): Texas Southwestern Medical Center 2003.
[50] Boonstra AM, Kooij JJ, Oosterlaan J, Sergeant JA, Buitelaar JK, Van Someren EJ. Hyperactive night and day? Actigraphy studies in adult ADHD: a baseline comparison and the effect of methylphenidate. Sleep 2007; 30: 433-442.

[51] Tuisku K, Virkkunen M, Holi M, Et A. Antisocial violent offenders with attention deficit hyperactivity disorder demonstrate akathisialike hyperactivity in three-channel actometry. J Neuropsych Clin N 2003; 15:194-9.

Received: March 12, 2012

Revised: June 02,2012

Accepted: June 25, 2012

(c) Edebol et al.; Licensee Bentham Open.

This is an open access article licensed under the terms of the Creative Commons Attribution Non-Commercial License (http://creativecommons.org/licenses/by-nc/3.0/) which permits unrestricted, non-commercial use, distribution and reproduction in any medium, provided the work is properly cited. 